\title{
Antiviral Therapy Use and Related Outcomes in Patients with Cancer and Viral Infections: Results from SWOG S1204
}

\author{
Jessica P. Hwang ( $\nabla$ jphwang@mdanderson.org ) \\ Kathryn B. Arnold \\ Fred Hutchinson Cancer Research Center \\ Joseph M. Unger \\ Fred Hutchinson Cancer Research Center \\ Rashmi Chugh \\ University of Michigan \\ Monica A. Tincopa \\ Centura Health \\ Rohit Loomba \\ University of California San Diego \\ Dawn Hershman \\ Columbia University \\ Scott D. Ramsey \\ Fred Hutchinson Cancer Research Center
}

The University of Texas MD Anderson Cancer Center https://orcid.org/0000-0002-5896-4182

\section{Research Article}

Keywords: hepatitis B virus, hepatitis C virus, HIV, latent infection, infection reactivation, antineoplastic agents

Posted Date: March 1st, 2022

DOI: https://doi.org/10.21203/rs.3.rs-1307960/v1

License: (c) (i) This work is licensed under a Creative Commons Attribution 4.0 International License. Read Full License 


\section{Abstract \\ Purpose}

Information is limited about adherence to practice guidelines in patients with hepatitis B virus (HBV), hepatitis C virus (HCV), or HIV infection receiving anticancer treatment.

\section{Methods}

Newly diagnosed adult cancer patients were enrolled in a multicenter, prospective cohort study (SWOG S1204) from 2013-2017 to evaluate the prevalence of HBV, HCV, or HIV in patients initiating anticancer treatment. At 6 months, records of virus-positive patients were reviewed for antiviral therapy use; anticancer treatment dose reduction; and HBV reactivation (elevated viral load). Categorical variables were compared using chi-square or Fisher's exact test.

\section{Results}

Of 3055 enrolled patients with viral testing, 230 had chronic or past HBV, HCV, or HIV with 6-month follow-up data (chronic HBV, 15 patients; past HBV, 158; HCV, 49; HIV, 30). Twenty percent (3/15) of chronic HBV and 11\% (17/158) of past HBV patients were co-infected with HCV and/or HIV. Rates of antiviral therapy use by 6 months were as follows: chronic HBV, 85\% (11/13); past HBV receiving anti-B cell therapy, $60 \%$ (3/5); past HBV receiving systemic anticancer therapy without anti-B cell therapy, 8\% (8/105); HCV, 6\% (2/35); and HIV, 90\% (19/21). Among patients with available data, anticancer treatment dose was reduced in 1 of 145 patients with past HBV and 1 of 42 with HCV. HBV reactivation occurred in 1 of 15 patients with chronic HBV; this patient was not receiving antiviral therapy.

\section{Conclusion}

In many patients with cancer and viral infections, antiviral treatment does not match guidelines. Further education is needed to improve adherence to guidelines.

\section{Introduction}

National organizations in the United States have published clinical practice guidelines for the care of patients with cancer and viral infections. According to the American Society of Clinical Oncology (ASCO) and National Comprehensive Cancer Network (NCCN) guidelines, patients with a hematologic malignancy and chronic hepatitis B virus (HBV) infection are recommended to start on anti-HBV therapy at the beginning of anticancer treatment to prevent reactivation and adverse liver outcomes.[1, 2] According to the ASCO and American Association for the Study of Liver Diseases (AASLD) guidelines, patients with a hematologic malignancy and past HBV infection who are anticipating stem cell transplant or anti-B cell therapy such as anti-CD20 monoclonal antibody drugs should also start on anti-HBV therapy at the beginning of anticancer treatment.[3, 1] An ASCO-supported clinical review[4] and the NCCN guidelines[2] recommend that patients with cancer and chronic hepatitis $\mathrm{C}$ virus $(\mathrm{HCV})$ infection should be treated with direct-acting antiviral therapy to prevent hepatitis flare and avoid delays in anticancer treatment, although NCCN consensus generally does not recommend concomitant HCV therapy during anticancer therapy.[2] The NCCN recommends that patients with cancer and HIV infection should be started on antiretroviral therapy to improve immune function and clinical outcomes.[5] The extent to which these clinical practice guidelines are followed is unknown. To address this knowledge gap, we examined antiviral therapy use along with anticancer treatment and virologic outcomes at 6 months in patients with cancer and HBV, HCV, or HIV infection enrolled in a large, multicenter, prospective cohort study of academic and community oncology practices across the United States.[6]

\section{Methods}

SWOG 1204 was a prospective observational study of patients at least 18 years of age who presented for initial treatment of a new malignancy at one of 18 participating academic or community oncology institutions during 2013-2017. The primary study findings were previously published.[6] Institutional review board approval for the primary study was provided by the Protocol Review Committee of the Cancer Therapy Evaluation Program on August 5, 2013. In brief, in the study, patients were categorized as positive or negative for chronic HBV, past HBV, HCV, or HIV infection based on standard diagnostic criteria for each virus, in order to establish the prevalence of these infections in cancer patients. Patients were categorized as positive for chronic HBV infection if they tested positive for hepatitis B surface antigen; positive for past HBV infection if they tested negative for hepatitis B surface antigen and positive for hepatitis B core antibody; positive for HCV infection if they had detectable HCV RNA; and positive for HIV if they had a documented history of HIV, positive HIV screening or confirmatory 
test, or detectable HIV RNA. The study estimated cancer population prevalence rates for past HBV, chronic HBV, HCV, and HIV infection in cancer patients at $5.3 \%, 0.4 \%, 1.9 \%$, and $1.0 \%$, respectively, and showed that most patients with past HBV and many with HCV infection were unaware of their positive viral status at cancer diagnosis. For all patients, HBV, HCV, and HIV status was determined either by documentation of viral status within 12 months prior to enrollment or by viral screening at the time of enrollment; more than $96 \%$ of patients had viral status determined within the three months prior to registration. Patients infected with more than 1 virus are included in the analysis for each infection, and no adjustments were made for co-infection.

Baseline data collected included cancer descriptors, planned treatment modalities, and laboratory values. For patients with HBV, HCV, or HIV infection, the participating study sites reviewed the medical records approximately 6 months after registration for the following postregistration events: most recent viral load; antiviral therapy received; type(s) of anticancer treatment received; changes in anticancer treatment due to viral infection (reduction in anticancer treatment dose or omission or suspension of anticancer treatment); and adverse liver outcomes due to viral infection (HBV reactivation [increase in HBV DNA], hepatitis flare [doubling of alanine transaminase or aspartate aminotransferase], increase in international normalized ratio [INR], or jaundice). Event dates were not collected. A patient could have more than one anticancer treatment modality, anticancer drug, change in cancer treatment, or adverse liver outcome reported.

This study extends the initial findings by examining antiviral therapy use, changes in anticancer treatment due to viral infection, and adverse liver outcomes due to viral infection. We compared observed antiviral management to guideline recommendations.[1-4] The rates of antiviral therapy use, changes in anticancer treatment due to viral infection, and adverse liver outcomes by type of viral infection were estimated among patients with known (non-missing) data. Descriptive statistics were used. The p-values for comparisons between categorical variables were obtained by using the chi-square test or Fisher's exact test, a t-test for continuous variables, and a Cochran-Armitage trend test for ordered variables. A two-sided alpha=.05 test was considered statistically significant. No adjustments were made for multiple comparisons.

\section{Results}

A total of 3055 newly diagnosed cancer patients who presented for treatment at one of the participating centers during the study period were registered to the study and had viral testing. Of these, 289 patients had viral infection; among these, 230 (80\%) had 6-month follow-up data available. There were no clinical significant differences in baseline patient characteristics between infected patients with and without 6-month data (summarized in Online Appendix Table). Among the patients with 6-month follow-up data available, 15 patients had chronic HBV, 158 had past HBV, 49 had HCV, and 30 had HIV infection (Figure 1). We found that 18 patients had 2 viruses, and 2 patients had 3 viruses. Among those with chronic HBV infection, 20\% (3/15) were co-infected (2 with HCV and 1 with HIV). Among patients with past HBV infection, 11\% (17/158) were co-infected (7 with HCV, 8 with HIV, and 2 with both HCV and HIV). No patients were co-infected with only HCV and HIV. Baseline characteristics of the patients with viral infection and 6-month follow-up data are presented in Table 1. 
Table 1

Demographic and Clinical Characteristics Overall and by Antiviral Therapy Use by 6 Months in Patients with HBV, HCV, or HIV Infection ${ }^{a, b}$

\begin{tabular}{|c|c|c|c|c|c|c|c|c|c|c|c|c|}
\hline \multirow[b]{3}{*}{ Characteristic } & \multicolumn{3}{|c|}{ Chronic HBV } & \multicolumn{3}{|l|}{ Past HBV } & \multicolumn{3}{|l|}{$\mathrm{HCV}$} & \multicolumn{3}{|l|}{ HIV } \\
\hline & \multirow[b]{2}{*}{$\begin{array}{l}\text { All } \\
\text { Patients }\end{array}$} & \multicolumn{2}{|c|}{$\begin{array}{l}\text { Anti-HBV } \\
\text { therapy use at } \\
6 \text { months }^{c}\end{array}$} & \multirow[b]{2}{*}{$\begin{array}{l}\text { All } \\
\text { Patients }\end{array}$} & \multicolumn{2}{|c|}{$\begin{array}{l}\text { Anti-HBV } \\
\text { therapy use at } \\
6 \text { months }\end{array}$} & \multirow[b]{2}{*}{$\begin{array}{l}\text { All } \\
\text { Patients }\end{array}$} & \multicolumn{2}{|c|}{$\begin{array}{l}\text { Anti-HCV } \\
\text { therapy use at } \\
6 \text { months }^{c}\end{array}$} & \multirow[b]{2}{*}{$\begin{array}{l}\text { All } \\
\text { Patients }\end{array}$} & \multicolumn{2}{|c|}{$\begin{array}{l}\text { Anti-HIV } \\
\text { therapy use at } \\
6 \text { months }^{c}\end{array}$} \\
\hline & & Yes & No & & Yes & No & & Yes & No & & Yes & No \\
\hline All patients & $\begin{array}{l}15 \\
(100)\end{array}$ & $\begin{array}{l}11 \\
(85)\end{array}$ & $\begin{array}{l}2 \\
(15)\end{array}$ & $\begin{array}{l}158 \\
(100)\end{array}$ & $\begin{array}{l}11 \\
(10)\end{array}$ & $\begin{array}{l}100 \\
(90)\end{array}$ & $\begin{array}{l}49 \\
(100)\end{array}$ & $2(6)$ & $\begin{array}{l}33 \\
(94)\end{array}$ & $\begin{array}{l}30 \\
(100)\end{array}$ & $\begin{array}{l}19 \\
(90)\end{array}$ & $\begin{array}{l}2 \\
(10)\end{array}$ \\
\hline $\begin{array}{l}\text { Age, median } \\
\text { (range), y }\end{array}$ & $\begin{array}{l}57.2 \\
(31.6- \\
68.0)\end{array}$ & $\begin{array}{l}57.3 \\
(31.6- \\
68.0)\end{array}$ & $\begin{array}{l}46.3 \\
(45.1- \\
47.5)\end{array}$ & $\begin{array}{l}61.0 \\
(27.9- \\
83.7)\end{array}$ & $\begin{array}{l}60.7 \\
(27.9- \\
73.0)\end{array}$ & $\begin{array}{l}61.0 \\
(29.9- \\
83.7)\end{array}$ & $\begin{array}{l}57.0 \\
(46.8- \\
77.5)\end{array}$ & $\begin{array}{l}63.8 \\
(54.4- \\
73.2)\end{array}$ & $\begin{array}{l}57.0 \\
(47.6- \\
77.5)\end{array}$ & $\begin{array}{l}52.8 \\
(21.8- \\
75.7)\end{array}$ & $\begin{array}{l}52.9 \\
(21.8- \\
69.4)\end{array}$ & $\begin{array}{l}40.0 \\
(34.8 \\
45.1)\end{array}$ \\
\hline \multicolumn{13}{|l|}{ Sex } \\
\hline Female & $4(27)$ & $\begin{array}{l}3 \\
(27)\end{array}$ & $0(0)$ & $91(58)$ & $\begin{array}{l}4 \\
(36)\end{array}$ & $\begin{array}{l}59 \\
(59)\end{array}$ & 19 (39) & $\begin{array}{l}1 \\
(50)\end{array}$ & $\begin{array}{l}16 \\
(48)\end{array}$ & $6(20)$ & $\begin{array}{l}3 \\
(16)\end{array}$ & $\begin{array}{l}1 \\
(50)\end{array}$ \\
\hline Male & $11(73)$ & $\begin{array}{l}8 \\
(73)\end{array}$ & $\begin{array}{l}2 \\
(100)\end{array}$ & $67(42)$ & $\begin{array}{l}7 \\
(64)\end{array}$ & $\begin{array}{l}41 \\
(41)\end{array}$ & $30(61)$ & $\begin{array}{l}1 \\
(50)\end{array}$ & $\begin{array}{l}17 \\
(52)\end{array}$ & $24(80)$ & $\begin{array}{l}16 \\
(84)\end{array}$ & $\begin{array}{l}1 \\
(50)\end{array}$ \\
\hline \multicolumn{13}{|l|}{ Race } \\
\hline White & $3(20)$ & $1(9)$ & $\begin{array}{l}1 \\
(50)\end{array}$ & 59 (37) & $\begin{array}{l}7 \\
(64)\end{array}$ & $\begin{array}{l}32 \\
(32)\end{array}$ & $28(57)$ & $\begin{array}{l}2 \\
(100)\end{array}$ & $\begin{array}{l}19 \\
(58)\end{array}$ & $16(53)$ & $\begin{array}{l}11 \\
(58)\end{array}$ & $\begin{array}{l}1 \\
(50)\end{array}$ \\
\hline Black & $5(33)$ & $\begin{array}{l}3 \\
(27)\end{array}$ & $\begin{array}{l}1 \\
(50)\end{array}$ & $58(37)$ & $0(0)$ & $\begin{array}{l}36 \\
(36)\end{array}$ & $17(35)$ & $0(0)$ & $\begin{array}{l}11 \\
(33)\end{array}$ & $13(43)$ & $\begin{array}{l}7 \\
(37)\end{array}$ & $\begin{array}{l}1 \\
(50)\end{array}$ \\
\hline Asian & $6(40)$ & $\begin{array}{l}6 \\
(55)\end{array}$ & $0(0)$ & $25(16)$ & $\begin{array}{l}4 \\
(36)\end{array}$ & $\begin{array}{l}19 \\
(19)\end{array}$ & $1(2)$ & $0(0)$ & $1(3)$ & $1(3)$ & $1(5)$ & $0(0)$ \\
\hline Other/multi-racial & $1(7)$ & $1(9)$ & $0(0)$ & $16(10)$ & $0(0)$ & $\begin{array}{l}13 \\
(13)\end{array}$ & $3(6)$ & $0(0)$ & $2(6)$ & $0(0)$ & $0(0)$ & $0(0)$ \\
\hline \multicolumn{13}{|l|}{ Ethnicity } \\
\hline Hispanic & $4(27)$ & $\begin{array}{l}3 \\
(27)\end{array}$ & $\begin{array}{l}1 \\
(50)\end{array}$ & 28 (18) & $1(9)$ & $\begin{array}{l}19 \\
(19)\end{array}$ & $6(13)$ & $0(0)$ & $\begin{array}{l}5 \\
(15)\end{array}$ & $6(20)$ & $\begin{array}{l}4 \\
(21)\end{array}$ & $\begin{array}{l}1 \\
(50)\end{array}$ \\
\hline Not Hispanic & $11(73)$ & $\begin{array}{l}8 \\
(73)\end{array}$ & $\begin{array}{l}1 \\
(50)\end{array}$ & $\begin{array}{l}129 \\
(82)\end{array}$ & $\begin{array}{l}10 \\
(91)\end{array}$ & $\begin{array}{l}80 \\
(81)\end{array}$ & $42(88)$ & $\begin{array}{l}1 \\
(100)\end{array}$ & $\begin{array}{l}28 \\
(85)\end{array}$ & $24(80)$ & $\begin{array}{l}15 \\
(79)\end{array}$ & $\begin{array}{l}1 \\
(50)\end{array}$ \\
\hline Unknown/missing & & & & 1 & & 1 & 1 & 1 & & & & \\
\hline \multicolumn{13}{|l|}{ Education } \\
\hline $\begin{array}{l}\text { Less than high } \\
\text { school diploma }\end{array}$ & $1(7)$ & $\begin{array}{l}1 \\
(10)\end{array}$ & $0(0)$ & 28 (19) & $0(0)$ & $\begin{array}{l}19 \\
(20)\end{array}$ & $15(31)$ & $0(0)$ & $\begin{array}{l}10 \\
(30)\end{array}$ & $7(23)$ & $\begin{array}{l}3 \\
(16)\end{array}$ & $\begin{array}{l}1 \\
(50)\end{array}$ \\
\hline $\begin{array}{l}\text { High school } \\
\text { diploma }\end{array}$ & $6(43)$ & $\begin{array}{l}3 \\
(30)\end{array}$ & $\begin{array}{l}1 \\
(50)\end{array}$ & $40(27)$ & $\begin{array}{l}3 \\
(27)\end{array}$ & $\begin{array}{l}23 \\
(24)\end{array}$ & $14(29)$ & $\begin{array}{l}1 \\
(50)\end{array}$ & $\begin{array}{l}7 \\
(21)\end{array}$ & $8(27)$ & $\begin{array}{l}6 \\
(32)\end{array}$ & $0(0)$ \\
\hline College & $7(50)$ & $\begin{array}{l}6 \\
(60)\end{array}$ & $\begin{array}{l}1 \\
(50)\end{array}$ & $68(45)$ & $\begin{array}{l}7 \\
(64)\end{array}$ & $\begin{array}{l}44 \\
(47)\end{array}$ & $16(33)$ & $0(0)$ & $\begin{array}{l}15 \\
(45)\end{array}$ & $11(37)$ & $\begin{array}{l}7 \\
(37)\end{array}$ & $\begin{array}{l}1 \\
(50)\end{array}$ \\
\hline Graduate school & $0(0)$ & $0(0)$ & $0(0)$ & $14(9)$ & $1(9)$ & $8(9)$ & $3(6)$ & $\begin{array}{l}1 \\
(50)\end{array}$ & $1(3)$ & $4(13)$ & $\begin{array}{l}3 \\
(16)\end{array}$ & $0(0)$ \\
\hline \multicolumn{13}{|c|}{ Abbreviations: HBV, hepatitis B virus; HCV, hepatitis C virus; GI, gastrointestinal. } \\
\hline \multicolumn{13}{|c|}{ aValues in table are number of patients (percentage) unless otherwise indicated. } \\
\hline \multicolumn{13}{|c|}{ bValues are measured at baseline except as noted. } \\
\hline \multicolumn{13}{|c|}{ 'Patients missing antiviral therapy status at 6 months are excluded; numbers do not add up across rows. } \\
\hline \multicolumn{13}{|c|}{ dSpecific to the virus indicated in the column heading, e.g., HBV for "Chronic HBV". } \\
\hline
\end{tabular}




\begin{tabular}{|c|c|c|c|c|c|c|c|c|c|c|c|c|}
\hline Missing & 1 & 1 & & 8 & & 6 & 1 & & & & & \\
\hline \multicolumn{13}{|l|}{ Employment } \\
\hline Employed & $6(43)$ & $\begin{array}{l}5 \\
(50)\end{array}$ & $0(0)$ & $48(31)$ & $\begin{array}{l}3 \\
(27)\end{array}$ & $\begin{array}{l}29 \\
(30)\end{array}$ & $12(25)$ & $0(0)$ & $\begin{array}{l}8 \\
(24)\end{array}$ & $8(28)$ & $\begin{array}{l}7 \\
(37)\end{array}$ & $0(0)$ \\
\hline Retired & $5(36)$ & $\begin{array}{l}4 \\
(40)\end{array}$ & $0(0)$ & $55(36)$ & $\begin{array}{l}2 \\
(18)\end{array}$ & $\begin{array}{l}36 \\
(37)\end{array}$ & $11(23)$ & $\begin{array}{l}1 \\
(50)\end{array}$ & $\begin{array}{l}8 \\
(24)\end{array}$ & $7(24)$ & $\begin{array}{l}4 \\
(21)\end{array}$ & $0(0)$ \\
\hline Unemployed & $1(7)$ & $\begin{array}{l}1 \\
(10)\end{array}$ & $0(0)$ & $24(16)$ & $\begin{array}{l}4 \\
(36)\end{array}$ & $\begin{array}{l}13 \\
(13)\end{array}$ & $12(25)$ & $\begin{array}{l}1 \\
(50)\end{array}$ & $\begin{array}{l}10 \\
(30)\end{array}$ & $9(31)$ & $\begin{array}{l}6 \\
(32)\end{array}$ & $\begin{array}{l}1 \\
(50)\end{array}$ \\
\hline Disabled or other & $2(14)$ & $0(0)$ & $\begin{array}{l}2 \\
(100)\end{array}$ & 27 (18) & $\begin{array}{l}2 \\
(18)\end{array}$ & $\begin{array}{l}19 \\
(20)\end{array}$ & $13(27)$ & $0(0)$ & $\begin{array}{l}7 \\
(21)\end{array}$ & $5(17)$ & $\begin{array}{l}2 \\
(11)\end{array}$ & $\begin{array}{l}1 \\
(50)\end{array}$ \\
\hline Missing & 1 & 1 & & 4 & & 3 & 1 & & & 1 & & \\
\hline \multicolumn{13}{|l|}{ Cancer type } \\
\hline $\begin{array}{l}\text { Hematologic } \\
\text { (any) }\end{array}$ & $1(7)$ & $1(9)$ & $0(0)$ & $13(8)$ & $\begin{array}{l}4 \\
(36)\end{array}$ & $7(7)$ & $8(16)$ & $0(0)$ & $\begin{array}{l}6 \\
(18)\end{array}$ & $7(23)$ & $\begin{array}{l}5 \\
(26)\end{array}$ & $\begin{array}{l}1 \\
(50)\end{array}$ \\
\hline Leukemia & $0(0 \%)$ & $\begin{array}{l}0 \\
(0 \%)\end{array}$ & $\begin{array}{l}0 \\
(0 \%)\end{array}$ & $1(1 \%)$ & $\begin{array}{l}0 \\
(0 \%)\end{array}$ & $\begin{array}{l}1 \\
(1 \%)\end{array}$ & $0(0 \%)$ & $\begin{array}{l}0 \\
(0 \%)\end{array}$ & $\begin{array}{l}0 \\
(0 \%)\end{array}$ & $1(3 \%)$ & $\begin{array}{l}1 \\
(5 \%)\end{array}$ & $\begin{array}{l}0 \\
(0 \%)\end{array}$ \\
\hline Lymphoma & $1(7 \%)$ & $\begin{array}{l}1 \\
(9 \%)\end{array}$ & $\begin{array}{l}0 \\
(0 \%)\end{array}$ & $2(1 \%)$ & $\begin{array}{l}0 \\
(0 \%)\end{array}$ & $\begin{array}{l}2 \\
(2 \%)\end{array}$ & $2(4 \%)$ & $\begin{array}{l}0 \\
(0 \%)\end{array}$ & $\begin{array}{l}2 \\
(6 \%)\end{array}$ & $0(0 \%)$ & $\begin{array}{l}0 \\
(0 \%)\end{array}$ & $\begin{array}{l}0 \\
(0 \%)\end{array}$ \\
\hline Myeloma & $0(0 \%)$ & $\begin{array}{l}0 \\
(0 \%)\end{array}$ & $\begin{array}{l}0 \\
(0 \%)\end{array}$ & $10(6 \%)$ & $\begin{array}{l}4 \\
(36 \%)\end{array}$ & $\begin{array}{l}4 \\
(4 \%)\end{array}$ & $6(12 \%)$ & $\begin{array}{l}0 \\
(0 \%)\end{array}$ & $\begin{array}{l}4 \\
(12 \%)\end{array}$ & $6(20 \%)$ & $\begin{array}{l}4 \\
(21 \%)\end{array}$ & $\begin{array}{l}1 \\
(50 \%)\end{array}$ \\
\hline Solid (any) & $14(93)$ & $\begin{array}{l}10 \\
(91)\end{array}$ & $\begin{array}{l}2 \\
(100)\end{array}$ & $\begin{array}{l}145 \\
(92)\end{array}$ & $\begin{array}{l}7 \\
(64)\end{array}$ & $\begin{array}{l}93 \\
(93)\end{array}$ & $41(84)$ & $\begin{array}{l}2 \\
(100)\end{array}$ & $\begin{array}{l}27 \\
(82)\end{array}$ & $23(77)$ & $\begin{array}{l}14 \\
(74)\end{array}$ & $\begin{array}{l}1 \\
(50)\end{array}$ \\
\hline Breast & $3(20 \%)$ & $\begin{array}{l}2 \\
(18 \%)\end{array}$ & $\begin{array}{l}0 \\
(0 \%)\end{array}$ & $\begin{array}{l}61 \\
(39 \%)\end{array}$ & $\begin{array}{l}1 \\
(9 \%)\end{array}$ & $\begin{array}{l}39 \\
(39 \%)\end{array}$ & $9(18 \%)$ & $\begin{array}{l}1 \\
(50 \%)\end{array}$ & $\begin{array}{l}7 \\
(21 \%)\end{array}$ & $2(7 \%)$ & $\begin{array}{l}2 \\
(11 \%)\end{array}$ & $\begin{array}{l}0 \\
(0 \%)\end{array}$ \\
\hline $\mathrm{Gl}$, colorectal & $1(7 \%)$ & $\begin{array}{l}1 \\
(9 \%)\end{array}$ & $\begin{array}{l}0 \\
(0 \%)\end{array}$ & $14(9 \%)$ & $\begin{array}{l}1 \\
(9 \%)\end{array}$ & $\begin{array}{l}9 \\
(9 \%)\end{array}$ & $6(12 \%)$ & $\begin{array}{l}0 \\
(0 \%)\end{array}$ & $\begin{array}{l}4 \\
(12 \%)\end{array}$ & $5(17 \%)$ & $\begin{array}{l}4 \\
(21 \%)\end{array}$ & $\begin{array}{l}0 \\
(0 \%)\end{array}$ \\
\hline GI, liver & $3(20 \%)$ & $\begin{array}{l}2 \\
(18 \%)\end{array}$ & $\begin{array}{l}1 \\
(50 \%)\end{array}$ & $3(2 \%)$ & $\begin{array}{l}0 \\
(0 \%)\end{array}$ & $\begin{array}{l}3 \\
(3 \%)\end{array}$ & $4(8 \%)$ & $\begin{array}{l}0 \\
(0 \%)\end{array}$ & $\begin{array}{l}4 \\
(12 \%)\end{array}$ & $0(0 \%)$ & $\begin{array}{l}0 \\
(0 \%)\end{array}$ & $\begin{array}{l}0 \\
(0 \%)\end{array}$ \\
\hline $\mathrm{Gl}$, other & $3(20 \%)$ & $\begin{array}{l}2 \\
(18 \%)\end{array}$ & $\begin{array}{l}1 \\
(50 \%)\end{array}$ & $\begin{array}{l}17 \\
(11 \%)\end{array}$ & $\begin{array}{l}1 \\
(9 \%)\end{array}$ & $\begin{array}{l}12 \\
(12 \%)\end{array}$ & $2(4 \%)$ & $\begin{array}{l}0 \\
(0 \%)\end{array}$ & $\begin{array}{l}1 \\
(3 \%)\end{array}$ & $5(17 \%)$ & $\begin{array}{l}2 \\
(11 \%)\end{array}$ & $\begin{array}{l}1 \\
(50 \%)\end{array}$ \\
\hline
\end{tabular}

Abbreviations: $\mathrm{HBV}$, hepatitis B virus; $\mathrm{HCV}$, hepatitis $\mathrm{C}$ virus; $\mathrm{Gl}$, gastrointestinal.

${ }^{a}$ Values in table are number of patients (percentage) unless otherwise indicated.

${ }^{b}$ Values are measured at baseline except as noted.

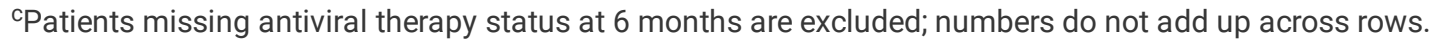

dSpecific to the virus indicated in the column heading, e.g., HBV for "Chronic HBV".

e Cancer treatment received by 6 months; may have received more than one type of treatment. 


\begin{tabular}{|c|c|c|c|c|c|c|c|c|c|c|c|c|}
\hline \multirow[b]{2}{*}{ Head and neck } & \multicolumn{3}{|c|}{ Chronic HBV } & \multicolumn{3}{|c|}{ Past HBV } & \multicolumn{3}{|l|}{$\mathrm{HCV}$} & \multicolumn{3}{|l|}{ HIV } \\
\hline & $1(7 \%)$ & $\begin{array}{l}1 \\
(9 \%)\end{array}$ & $\begin{array}{l}0 \\
(0 \%)\end{array}$ & $8(5 \%)$ & $\begin{array}{l}0 \\
(0 \%)\end{array}$ & $\begin{array}{l}6 \\
(6 \%)\end{array}$ & $5(10 \%)$ & $\begin{array}{l}0 \\
(0 \%)\end{array}$ & $\begin{array}{l}4 \\
(12 \%)\end{array}$ & $1(3 \%)$ & $\begin{array}{l}1 \\
(5 \%)\end{array}$ & $\begin{array}{l}0 \\
(0 \%)\end{array}$ \\
\hline Lung & $2(13 \%)$ & $\begin{array}{l}1 \\
(9 \%)\end{array}$ & $\begin{array}{l}0 \\
(0 \%)\end{array}$ & $\begin{array}{l}19 \\
(12 \%)\end{array}$ & $\begin{array}{l}2 \\
(18 \%)\end{array}$ & $\begin{array}{l}12 \\
(12 \%)\end{array}$ & $8(16 \%)$ & $\begin{array}{l}0 \\
(0 \%)\end{array}$ & $\begin{array}{l}2 \\
(6 \%)\end{array}$ & $1(3 \%)$ & $\begin{array}{l}1 \\
(5 \%)\end{array}$ & $\begin{array}{l}0 \\
(0 \%)\end{array}$ \\
\hline Prostate & $0(0 \%)$ & $\begin{array}{l}0 \\
(0 \%)\end{array}$ & $\begin{array}{l}0 \\
(0 \%)\end{array}$ & $9(6 \%)$ & $\begin{array}{l}0 \\
(0 \%)\end{array}$ & $\begin{array}{l}3 \\
(3 \%)\end{array}$ & $3(6 \%)$ & $\begin{array}{l}1 \\
(50 \%)\end{array}$ & $\begin{array}{l}1 \\
(3 \%)\end{array}$ & $1(3 \%)$ & $\begin{array}{l}0 \\
(0 \%)\end{array}$ & $\begin{array}{l}0 \\
(0 \%)\end{array}$ \\
\hline Other & $1(7 \%)$ & $\begin{array}{l}1 \\
(9 \%)\end{array}$ & $\begin{array}{l}0 \\
(0 \%)\end{array}$ & $14(9 \%)$ & $\begin{array}{l}2 \\
(18 \%)\end{array}$ & $\begin{array}{l}9 \\
(9 \%)\end{array}$ & $4(8 \%)$ & $\begin{array}{l}0 \\
(0 \%)\end{array}$ & $\begin{array}{l}4 \\
(12 \%)\end{array}$ & $8(27 \%)$ & $\begin{array}{l}4 \\
(21 \%)\end{array}$ & $\begin{array}{l}0 \\
(0 \%)\end{array}$ \\
\hline \multicolumn{13}{|l|}{$\begin{array}{l}\text { Performance } \\
\text { status (Zubrod) }\end{array}$} \\
\hline 0 & $5(42 \%)$ & $\begin{array}{l}3 \\
(38 \%)\end{array}$ & $\begin{array}{l}1 \\
(50 \%)\end{array}$ & $\begin{array}{l}66 \\
(52 \%)\end{array}$ & $\begin{array}{l}4 \\
(44 \%)\end{array}$ & $\begin{array}{l}42 \\
(52 \%)\end{array}$ & $\begin{array}{l}23 \\
(51 \%)\end{array}$ & $\begin{array}{l}1 \\
(50 \%)\end{array}$ & $\begin{array}{l}15 \\
(52 \%)\end{array}$ & $\begin{array}{l}12 \\
(50 \%)\end{array}$ & $\begin{array}{l}8 \\
(57 \%)\end{array}$ & $\begin{array}{l}1 \\
(50 \%)\end{array}$ \\
\hline 1 & $6(50 \%)$ & $\begin{array}{l}4 \\
(50 \%)\end{array}$ & $\begin{array}{l}1 \\
(50 \%)\end{array}$ & $\begin{array}{l}54 \\
(42 \%)\end{array}$ & $\begin{array}{l}4 \\
(44 \%)\end{array}$ & $\begin{array}{l}33 \\
(41 \%)\end{array}$ & $\begin{array}{l}16 \\
(36 \%)\end{array}$ & $\begin{array}{l}1 \\
(50 \%)\end{array}$ & $\begin{array}{l}10 \\
(34 \%)\end{array}$ & $\begin{array}{l}10 \\
(42 \%)\end{array}$ & $\begin{array}{l}5 \\
(36 \%)\end{array}$ & $\begin{array}{l}1 \\
(50 \%)\end{array}$ \\
\hline 2 & $1(8 \%)$ & $\begin{array}{l}1 \\
(13 \%)\end{array}$ & $\begin{array}{l}0 \\
(0 \%)\end{array}$ & $6(5 \%)$ & $\begin{array}{l}1 \\
(11 \%)\end{array}$ & $\begin{array}{l}5 \\
(6 \%)\end{array}$ & $4(9 \%)$ & $\begin{array}{l}0 \\
(0 \%)\end{array}$ & $\begin{array}{l}3 \\
(10 \%)\end{array}$ & $0(0 \%)$ & $\begin{array}{l}0 \\
(0 \%)\end{array}$ & $\begin{array}{l}0 \\
(0 \%)\end{array}$ \\
\hline 3 & $0(0 \%)$ & $\begin{array}{l}0 \\
(0 \%)\end{array}$ & $\begin{array}{l}0 \\
(0 \%)\end{array}$ & $2(2 \%)$ & $\begin{array}{l}0 \\
(0 \%)\end{array}$ & $\begin{array}{l}1 \\
(1 \%)\end{array}$ & $2(4 \%)$ & $\begin{array}{l}0 \\
(0 \%)\end{array}$ & $\begin{array}{l}1 \\
(3 \%)\end{array}$ & $2(8 \%)$ & $\begin{array}{l}1 \\
(7 \%)\end{array}$ & $\begin{array}{l}0 \\
(0 \%)\end{array}$ \\
\hline Missing & 3 & 3 & & 30 & 2 & 19 & 4 & & 4 & 6 & 5 & \\
\hline \multicolumn{13}{|l|}{$\begin{array}{l}\text { Prior viral } \\
\text { diagnosis }^{\mathrm{d}}\end{array}$} \\
\hline Yes & $9(60)$ & $\begin{array}{l}8 \\
(73)\end{array}$ & $\begin{array}{l}1 \\
(50)\end{array}$ & $22(14)$ & $\begin{array}{l}4 \\
(36)\end{array}$ & $\begin{array}{l}16 \\
(16)\end{array}$ & $34(69)$ & $\begin{array}{l}2 \\
(100)\end{array}$ & $\begin{array}{l}24 \\
(73)\end{array}$ & $29(97)$ & $\begin{array}{l}19 \\
(100)\end{array}$ & $\begin{array}{l}1 \\
(50)\end{array}$ \\
\hline No & $4(27)$ & $\begin{array}{l}2 \\
(18)\end{array}$ & $\begin{array}{l}1 \\
(50)\end{array}$ & $\begin{array}{l}110 \\
(70)\end{array}$ & $\begin{array}{l}4 \\
(36)\end{array}$ & $\begin{array}{l}69 \\
(69)\end{array}$ & $12(24)$ & $0(0)$ & $\begin{array}{l}8 \\
(24)\end{array}$ & $1(3)$ & $0(0)$ & $\begin{array}{l}1 \\
(50)\end{array}$ \\
\hline Don't know & $2(13)$ & $1(9)$ & $0(0)$ & $26(16)$ & $\begin{array}{l}3 \\
(27)\end{array}$ & $\begin{array}{l}15 \\
(15)\end{array}$ & $3(6)$ & $0(0)$ & $1(3)$ & $0(0)$ & $0(0)$ & $0(0)$ \\
\hline \multicolumn{13}{|l|}{ Viral loadd } \\
\hline Detectable & $4(27)$ & $\begin{array}{l}4 \\
(36)\end{array}$ & $0(0)$ & $0(0)$ & $0(0)$ & $0(0)$ & $\begin{array}{l}49 \\
(100)\end{array}$ & $\begin{array}{l}2 \\
(100)\end{array}$ & $\begin{array}{l}33 \\
(100)\end{array}$ & $11(37)$ & $\begin{array}{l}7 \\
(37)\end{array}$ & $\begin{array}{l}1 \\
(50)\end{array}$ \\
\hline Undetectable & $2(13)$ & $\begin{array}{l}2 \\
(18)\end{array}$ & $0(0)$ & $16(10)$ & $\begin{array}{l}2 \\
(18)\end{array}$ & $\begin{array}{l}10 \\
(10)\end{array}$ & $0(0)$ & $0(0)$ & $0(0)$ & $14(47)$ & $\begin{array}{l}10 \\
(53)\end{array}$ & $\begin{array}{l}1 \\
(50)\end{array}$ \\
\hline Not done/reported & $9(60)$ & $\begin{array}{l}5 \\
(45)\end{array}$ & $\begin{array}{l}2 \\
(100)\end{array}$ & $\begin{array}{l}142 \\
(90)\end{array}$ & $\begin{array}{l}9 \\
(82)\end{array}$ & $\begin{array}{l}90 \\
(90)\end{array}$ & $0(0)$ & $0(0)$ & $0(0)$ & $5(17)$ & $\begin{array}{l}2 \\
(11)\end{array}$ & $0(0)$ \\
\hline \multicolumn{13}{|l|}{ Cancer treatment $\mathrm{e}^{\mathrm{e}}$} \\
\hline $\begin{array}{l}\text { Cytotoxic } \\
\text { chemotherapy }\end{array}$ & $\begin{array}{l}11 \\
(79 \%)\end{array}$ & $\begin{array}{l}9 \\
(82 \%)\end{array}$ & $\begin{array}{l}1 \\
(50 \%)\end{array}$ & $\begin{array}{l}113 \\
(73 \%)\end{array}$ & $\begin{array}{l}9 \\
(82 \%)\end{array}$ & $\begin{array}{l}72 \\
(72 \%)\end{array}$ & $\begin{array}{l}36 \\
(77 \%)\end{array}$ & $\begin{array}{l}1 \\
(50 \%)\end{array}$ & $\begin{array}{l}26 \\
(79 \%)\end{array}$ & $\begin{array}{l}20 \\
(74 \%)\end{array}$ & $\begin{array}{l}15 \\
(79 \%)\end{array}$ & $\begin{array}{l}1 \\
(50 \%)\end{array}$ \\
\hline Immunotherapy & $0(0 \%)$ & $\begin{array}{l}0 \\
(0 \%)\end{array}$ & $\begin{array}{l}0 \\
(0 \%)\end{array}$ & $4(3 \%)$ & $\begin{array}{l}0 \\
(0 \%)\end{array}$ & $\begin{array}{l}4 \\
(4 \%)\end{array}$ & $2(4 \%)$ & $\begin{array}{l}0 \\
(0 \%)\end{array}$ & $\begin{array}{l}1 \\
(3 \%)\end{array}$ & $0(0 \%)$ & $\begin{array}{l}0 \\
(0 \%)\end{array}$ & $\begin{array}{l}0 \\
(0 \%)\end{array}$ \\
\hline
\end{tabular}

Abbreviations: $\mathrm{HBV}$, hepatitis B virus; $\mathrm{HCV}$, hepatitis $\mathrm{C}$ virus; $\mathrm{Gl}$, gastrointestinal.

${ }^{a}$ Values in table are number of patients (percentage) unless otherwise indicated.

${ }^{b}$ Values are measured at baseline except as noted.

'Patients missing antiviral therapy status at 6 months are excluded; numbers do not add up across rows.

dSpecific to the virus indicated in the column heading, e.g., HBV for "Chronic HBV".

e Cancer treatment received by 6 months; may have received more than one type of treatment. 


\begin{tabular}{|c|c|c|c|c|c|c|c|c|c|c|c|c|}
\hline & Chronic H & & & Past HBV & & & $\mathrm{HCV}$ & & & HIV & & \\
\hline $\begin{array}{l}\text { Targeted biologic } \\
\text { drug }\end{array}$ & $1(7 \%)$ & $\begin{array}{l}1 \\
(9 \%)\end{array}$ & $\begin{array}{l}0 \\
(0 \%)\end{array}$ & $10(6 \%)$ & $\begin{array}{l}4 \\
(36 \%)\end{array}$ & $\begin{array}{l}4 \\
(4 \%)\end{array}$ & $1(2 \%)$ & $\begin{array}{l}0 \\
(0 \%)\end{array}$ & $\begin{array}{l}1 \\
(3 \%)\end{array}$ & $1(4 \%)$ & $\begin{array}{l}1 \\
(5 \%)\end{array}$ & $\begin{array}{l}0 \\
(0 \%)\end{array}$ \\
\hline $\begin{array}{l}\text { Bone marrow } \\
\text { transplant }\end{array}$ & $0(0 \%)$ & $\begin{array}{l}0 \\
(0 \%)\end{array}$ & $\begin{array}{l}0 \\
(0 \%)\end{array}$ & $0(0 \%)$ & $\begin{array}{l}0 \\
(0 \%)\end{array}$ & $\begin{array}{l}0 \\
(0 \%)\end{array}$ & $1(2 \%)$ & $\begin{array}{l}0 \\
(0 \%)\end{array}$ & $\begin{array}{l}1 \\
(3 \%)\end{array}$ & 0 (0\%) & $\begin{array}{l}0 \\
(0 \%)\end{array}$ & $\begin{array}{l}0 \\
(0 \%)\end{array}$ \\
\hline $\begin{array}{l}\text { Anti-B cell therapy, } \\
\text { including } \\
\text { rituximab }\end{array}$ & $0(0 \%)$ & $\begin{array}{l}0 \\
(0 \%)\end{array}$ & $\begin{array}{l}0 \\
(0 \%)\end{array}$ & $6(4 \%)$ & $\begin{array}{l}3 \\
(27 \%)\end{array}$ & $\begin{array}{l}2 \\
(2 \%)\end{array}$ & $5(11 \%)$ & $\begin{array}{l}0 \\
(0 \%)\end{array}$ & $\begin{array}{l}5 \\
(15 \%)\end{array}$ & $2(7 \%)$ & $\begin{array}{l}2 \\
(11 \%)\end{array}$ & $\begin{array}{l}0 \\
(0 \%)\end{array}$ \\
\hline Radiation therapy & $5(36 \%)$ & $\begin{array}{l}3 \\
(27 \%)\end{array}$ & $\begin{array}{l}1 \\
(50 \%)\end{array}$ & $\begin{array}{l}67 \\
(43 \%)\end{array}$ & $\begin{array}{l}1 \\
(9 \%)\end{array}$ & $\begin{array}{l}47 \\
(47 \%)\end{array}$ & $\begin{array}{l}14 \\
(30 \%)\end{array}$ & $\begin{array}{l}1 \\
(50 \%)\end{array}$ & $\begin{array}{l}10 \\
(30 \%)\end{array}$ & $9(33 \%)$ & $\begin{array}{l}5 \\
(26 \%)\end{array}$ & $\begin{array}{l}1 \\
(50 \%)\end{array}$ \\
\hline Surgery & $2(14 \%)$ & $\begin{array}{l}2 \\
(18 \%)\end{array}$ & $\begin{array}{l}0 \\
(0 \%)\end{array}$ & $\begin{array}{l}48 \\
(31 \%)\end{array}$ & $\begin{array}{l}2 \\
(18 \%)\end{array}$ & $\begin{array}{l}37 \\
(37 \%)\end{array}$ & $5(11 \%)$ & $\begin{array}{l}0 \\
(0 \%)\end{array}$ & $\begin{array}{l}4 \\
(12 \%)\end{array}$ & $4(15 \%)$ & $\begin{array}{l}3 \\
(16 \%)\end{array}$ & $\begin{array}{l}0 \\
(0 \%)\end{array}$ \\
\hline $\begin{array}{l}\text { Treatment } \\
\text { unknown }\end{array}$ & $0(0 \%)$ & $\begin{array}{l}0 \\
(0 \%)\end{array}$ & $\begin{array}{l}0 \\
(0 \%)\end{array}$ & $1(1 \%)$ & $\begin{array}{l}0 \\
(0 \%)\end{array}$ & $\begin{array}{l}1 \\
(1 \%)\end{array}$ & $1(2 \%)$ & $\begin{array}{l}0 \\
(0 \%)\end{array}$ & $\begin{array}{l}0 \\
(0 \%)\end{array}$ & $0(0 \%)$ & $\begin{array}{l}0 \\
(0 \%)\end{array}$ & $\begin{array}{l}0 \\
(0 \%)\end{array}$ \\
\hline $\begin{array}{l}\text { Missing treatment } \\
\text { data }\end{array}$ & 1 & & & 3 & & & 2 & & & 3 & & \\
\hline \multicolumn{13}{|c|}{ Abbreviations: HBV, hepatitis B virus; HCV, hepatitis C virus; GI, gastrointestinal. } \\
\hline \multicolumn{13}{|c|}{ avalues in table are number of patients (percentage) unless otherwise indicated. } \\
\hline \multicolumn{13}{|c|}{ bValues are measured at baseline except as noted. } \\
\hline \multicolumn{13}{|c|}{ 'Patients missing antiviral therapy status at 6 months are excluded; numbers do not add up across rows. } \\
\hline \multicolumn{13}{|c|}{ dSpecific to the virus indicated in the column heading, e.g., HBV for "Chronic HBV". } \\
\hline
\end{tabular}

\section{Chronic HBV infection}

Of 3054 newly diagnosed cancer patients evaluated for HBV infection, 19 (1\%) had chronic HBV infection (Figure 1). Of these 19 patients, 15 (79\%) had 6-month follow-up data available (Online-Only Appendix Table).

Among patients with known chronic HBV infection whose medication status was available, 15\% (2/13) had not received anti-HBV medication by 6 months (Table 1). Those who received anti-HBV medication received entecavir, tenofovir disoproxil fumarate, or lamivudine. One patient with chronic HBV infection had an adverse liver outcome, HBV reactivation; this patient had not received anti-HBV therapy by 6 months (Table 2). One patient with chronic HBV infection had a hematologic malignancy (Table 1); this patient received antiviral therapy. There were no cases of change in anticancer treatment due to viral infection in patients with chronic HBV infection (Table 3). 
Table 2

Antiviral Therapy Use among Patients with Known Adverse Liver Outcomes by 6 Months in Patients with HBV, HCV, or HIV Infection ${ }^{a}$

\begin{tabular}{|c|c|c|c|c|c|c|c|c|c|c|c|c|}
\hline & \multicolumn{3}{|c|}{ Chronic HBV } & \multicolumn{3}{|l|}{ Past HBV } & \multicolumn{3}{|l|}{$\mathrm{HCV}$} & \multicolumn{3}{|l|}{ HIV } \\
\hline & & \multicolumn{2}{|c|}{$\begin{array}{l}\text { Anti-HBV } \\
\text { Therapy Use at } 6 \\
\text { Months }^{b}\end{array}$} & & \multicolumn{2}{|c|}{$\begin{array}{l}\text { Anti-HBV } \\
\text { Therapy Use at } 6 \\
\text { Months }^{b}\end{array}$} & \multirow[b]{2}{*}{$\begin{array}{l}\text { All } \\
\text { Patients }\end{array}$} & \multicolumn{2}{|c|}{$\begin{array}{l}\text { Anti-HCV } \\
\text { Therapy Use at } \\
6 \text { Months }^{b}\end{array}$} & \multirow[b]{2}{*}{$\begin{array}{l}\text { All } \\
\text { Patients }\end{array}$} & \multicolumn{2}{|c|}{$\begin{array}{l}\text { Anti-HIV } \\
\text { Therapy } \\
\text { Use at } 6 \\
\text { Months }^{b}\end{array}$} \\
\hline & $\begin{array}{l}\text { All } \\
\text { Patients }\end{array}$ & Yes & No & $\begin{array}{l}\text { All } \\
\text { Patients }\end{array}$ & Yes & No & & Yes & No & & Yes & No \\
\hline $\begin{array}{l}\text { Any adverse } \\
\text { liver outcome }\end{array}$ & $1(100)$ & $\begin{array}{l}0 / 1 \\
(0)\end{array}$ & $\begin{array}{l}1 / 1 \\
(100)\end{array}$ & $\begin{array}{l}29 \\
(100)\end{array}$ & $\begin{array}{l}5 / 24 \\
(21)\end{array}$ & $\begin{array}{l}19 / 24 \\
(79)\end{array}$ & $\begin{array}{l}10 \\
(100)\end{array}$ & $\begin{array}{l}1 / 8 \\
(13)\end{array}$ & $\begin{array}{l}7 / 8 \\
(87)\end{array}$ & $5(100)$ & $\begin{array}{l}4 / 4 \\
(100)\end{array}$ & $\begin{array}{l}0 / 4 \\
(0)\end{array}$ \\
\hline \multicolumn{13}{|l|}{$\begin{array}{l}\text { Specific } \\
\text { adverse liver } \\
\text { outcomes }^{c}\end{array}$} \\
\hline $\begin{array}{l}\mathrm{HBV} \\
\text { reactivation }\end{array}$ & $1(100)$ & - & $1(100)$ & $0(0)$ & $0(0)$ & $0(0)$ & $0(0)$ & $0(0)$ & $0(0)$ & $0(0)$ & $0(0)$ & - \\
\hline Hepatitis flare & $0(0)$ & - & $0(0)$ & $23(79)$ & $\begin{array}{l}5 \\
(100)\end{array}$ & $14(74)$ & $7(70)$ & 1 (100) & $5(71)$ & $5(100)$ & $\begin{array}{l}4 \\
(100)\end{array}$ & - \\
\hline Increase in INR & $0(0)$ & - & $0(0)$ & $12(41)$ & $1(20)$ & $9(47)$ & $5(50)$ & $0(0)$ & $4(57)$ & $1(20)$ & $\begin{array}{l}1 \\
(25)\end{array}$ & - \\
\hline $\begin{array}{l}\text { Developed } \\
\text { jaundice }\end{array}$ & $0(0)$ & - & $0(0)$ & $1(3)$ & $0(0)$ & $1(5)$ & $3(30)$ & $\begin{array}{l}1 \\
(100)\end{array}$ & $1(14)$ & $0(0)$ & $0(0)$ & - \\
\hline \multicolumn{13}{|c|}{ Abbreviations: HBV, hepatitis B virus; HCV, hepatitis C virus; INR, international normalized ratio. } \\
\hline \multicolumn{13}{|c|}{ avalues in table are number of patients (percentage). } \\
\hline \multicolumn{13}{|c|}{$\begin{array}{l}\text { bPatients missing antiviral therapy status at } 6 \text { months are excluded; the denominators show the numbers of patients for whom antiviral } \\
\text { status was available. }\end{array}$} \\
\hline
\end{tabular}


Table 3

Viral-Related Changes in Treatment by 6 Months in Patients with HBV, HCV, or HIV Infection ${ }^{\text {a }}$

\begin{tabular}{|c|c|c|c|c|}
\hline & $\begin{array}{l}\text { Chronic HBV } \\
(n=15)\end{array}$ & $\begin{array}{l}\text { Past HBV } \\
(n=158)\end{array}$ & $\begin{array}{l}\text { HCV } \\
(n=49)\end{array}$ & $\begin{array}{l}\text { HIV } \\
(n=30)\end{array}$ \\
\hline \multicolumn{5}{|l|}{ Change in treatment due to viral infection } \\
\hline Yes & $2(14)$ & $12(8)$ & $3(7)$ & $5(21)$ \\
\hline No & $12(86)$ & $134(92)$ & $42(93)$ & $19(79)$ \\
\hline Unknown/missing & 1 & 12 & 4 & 6 \\
\hline \multicolumn{5}{|l|}{ Type of change ${ }^{b}$} \\
\hline Reduction of chemotherapy dose & $0(0)$ & $0(0)$ & $0(0)$ & $0(0)$ \\
\hline Omission of anticancer treatment due to viral infection & $0(0)$ & $0(0)$ & $0(0)$ & $0(0)$ \\
\hline Addition of antiviral/antiretroviral therapy & $2(14)$ & $8(5)$ & $1(2)$ & $4(17)$ \\
\hline Other change & $0(0)$ & $6(4)$ & $2(4)$ & $1(4)$ \\
\hline \multicolumn{5}{|c|}{ Change in treatment due to end organ effects of viral infection } \\
\hline Yes & $1(7)$ & $4(3)$ & $2(5)$ & $1(4)$ \\
\hline No & $13(93)$ & $141(97)$ & $40(95)$ & $25(96)$ \\
\hline Unknown/missing & 1 & 13 & 7 & 4 \\
\hline \multicolumn{5}{|l|}{ Type of change } \\
\hline Reduction of chemotherapy dose & $0(0)$ & $1(1)$ & $1(2)$ & $0(0)$ \\
\hline Omission of anticancer treatment due to viral infection & $0(0)$ & $0(0)$ & $1(2)$ & $0(0)$ \\
\hline Addition of antiviral/antiretroviral therapy & $1(7)$ & $2(1)$ & $0(0)$ & $1(4)$ \\
\hline Other change & $0(0)$ & $1(1)$ & $0(0)$ & $0(0)$ \\
\hline \multicolumn{5}{|l|}{ Abbreviations: HBV, hepatitis B virus; HCV, hepatitis C virus. } \\
\hline \multicolumn{5}{|l|}{${ }^{a}$ Values in table are number of patients (percentage). } \\
\hline
\end{tabular}

\section{Past HBV infection}

Of 3054 newly diagnosed cancer patients evaluated for HBV infection, 198 (6\%) had past HBV infection (Figure 1). Of these 198 patients, 158 (80\%) had 6-month follow-up data available (Online-Only Appendix Table).

Among patients with known past HBV infection whose medication status was available, 10\% (11/111) received antiviral therapy by 6 months (Table 1). Those who received anti-HBV medication received entecavir, tenofovir disoproxil fumarate, or lamivudine. Compared to patients who had not received anti-HBV medication by 6 months, patients who had received anti-HBV medication by 6 months had higher rates of white race, non-Hispanic ethnicity, male sex, unemployed status, high education level, and hematologic cancers (Table 1). Among patients with past HBV infection receiving anti-B cell therapy, 40\% (2/5) had not received antiviral therapy by 6 months. Taking into account that combinations of anticancer drugs were possible, 50\% (4/8) of patients with past HBV infection receiving targeted biologic therapy, $11 \%(9 / 81)$ receiving cytotoxic therapy, and 8\% (8/105) receiving systemic anticancer therapy without an anti-B cell drug received antiviral therapy by 6 months.

Twenty-nine patients with past HBV infection had at least one adverse liver outcome: 23 (79\%) had a hepatitis flare, 12 (41\%) had an increase in INR, and 1 (3\%) had jaundice (Table 2). Fourteen patients with past HBV infection and hepatitis flare had not received anti-HBV therapy by 6 months (Table 2). One patient (1\%) with past HBV infection required a lowering of chemotherapy dose (Table 3 ).

\section{HCV infection}

Of 2994 newly diagnosed cancer patients evaluated for HCV infection, 70 (2\%) were infected with HCV (Figure 1). Of these 70 patients, 49 (70\%) had 6-month follow-up data available (Online-Only Appendix Table). 
Among patients with known HCV infection whose medication status was available, 94\% (33/35) had not received anti-HCV medication by 6 months (Table 1). Those who were treated with anti-HCV medications received ledipasvir/sofosbuvir combination. Ten patients with HCV infection had at least one adverse liver outcomes: 7 (70\%) had a hepatitis flare, 5 (50\%) had an increase in INR, and 3 (30\%) had jaundice (Table 2). Five patients with HCV infection and hepatitis flare had not received anti-HCV therapy by 6 months (Table 2). Three patients (3/33, $9 \%)$ had changes in treatment possibly due to viral infection (Table 3$)$. Two patients $(2 / 42,5 \%)$ had a change in anticancer treatment due to end organ effects associated with viral infection: one had the chemotherapy dose lowered, and one had therapy omitted that would have been recommended had the patient's viral status been negative (Table 3).

\section{HIV infection}

Of 3049 newly diagnosed cancer patients evaluated for HIV infection, 34 (1\%) were infected with HIV (Figure 1). Of these 34 patients, 30 (88\%) had 6-month follow-up data available (Online-Only Appendix Table).

Among patients with known HIV infection whose medication status was available, 10\% (2/21) had not received anti-HIV medications by 6 months (Table 1). Those who were treated for HIV received the following medications: nucleoside reverse transcriptase inhibitors (abacavir, emtricitabine, lamivudine, tenofovir disoproxil fumarate, or zidovudine); non-nucleoside reverse transcriptase inhibitors (efavirenz, etravirine, or nevirapine); protease inhibitors (ritonavir or darunavir); integrase strand transfer inhibitors (raltegravir or dolutegravir); or a fixed combination therapy (efavirenz, emtricitabine, and tenofovir disoproxil fumarate; lamivudine and zidovudine; abacavir and lamivudine; emtricitabine and tenofovir disoproxil fumarate; darunavir and cobicistat; or abacavir, dolutegravir, and lamivudine). Five patients with HIV infection had an adverse liver outcome: 5 (100\%) had a hepatitis flare, and 1 (20\%) had an increase in INR (Table 2). All 4 patients with HIV infection and hepatitis flare for whom data were available had received anti-HIV therapy by 6 months (Table 2). No patient with HIV infection had a lowered dose of chemotherapy or omission of an anticancer drug (Table 3).

\section{Discussion}

In this study, we examined the patterns of use of antiviral therapy, the rates of changes in anticancer treatment due to viral infection, and adverse liver outcomes at 6 months in patients with cancer and HBV, HCV, or HIV infection. Though ASCO,[1] AASLD,[3] and NCCN[2] guidelines recommend that patients with chronic HBV infection and NCCN guidelines[5] recommend that patients with HIV infection receiving anticancer treatment receive concomitant antiviral therapy, we found that $15 \%$ of cancer patients with chronic HBV infection and $10 \%$ of cancer patients with HIV infection had not received antivirals by 6 months. Further, while ASCO guidelines[1] recommend that patients with past HBV infection treated with anti-CD20 drugs receive concomitant antiviral prophylaxis, 40\% (2/5) of our patients with past HBV infection treated with antiCD20 therapy had not received antiviral therapy by 6 months. On the other hand, $8 \%$ of patients with past HBV infection who received anticancer treatment that did not include an anti-CD20 drug-a group for which monitoring without antiviral prophylaxis is recommended by ASCO[1] and AASLD[3]-actually had received antiviral therapy by 6 months. Although experts recommend antiviral therapy for cancer patients with HCV infection, $[2,4]$ only $6 \%$ of our patients with HCV infection had received antiviral therapy by 6 months.

For patients with chronic HBV infection and a hematologic malignancy, in whom the risk of HBV reactivation is nearly 50\%,[7] ASCO,[1] NCCN, [2] AASLD[3] guidelines recommend antiviral prophylaxis prior to anticancer treatment. In this study, the sole patient with a hematologic malignancy and chronic HBV infection did receive antiviral prophylaxis and had no adverse liver outcomes or changes in anticancer treatment. It is estimated that the risk of reactivation for patients with solid tumors and chronic HBV infection is 25\%.[8] However, because the risk of reactivation during treatment with individual anticancer drugs or combinations of anticancer drugs is unclear for patients with solid tumors, the optimal timing for antiviral therapy for patients with solid tumors and chronic HBV infection has not been determined,[1] and treatment options may include antiviral prophylaxis at the initiation of anticancer treatment or close monitoring with initiation of antiviral therapy at the earliest sign of HBV reactivation.

Patients with past HBV infection receiving anti-B cell therapy, such as anti-CD20 monoclonal antibody, are at high risk of HBV reactivation and should receive antiviral prophylaxis, as recommended by ASCO and AASLD.[1, 3] Patients with past HBV infection have covalently closed circular DNA capable of replication remaining in the hepatocytes. Replication is normally inhibited by a healthy host's strong immune system but may spiral out of control and lead to HBV reactivation with potent immunosuppression. In our study, we found that $40 \%$ (2/5) of the patients with past HBV infection receiving anti-B cell therapy had not received antiviral prophylaxis by 6 months; none of these patients had developed HBV reactivation by 6 months.

On the other hand, the risk of HBV reactivation is low for patients with a solid tumor and past HBV infection receiving anticancer treatment that does not include anti-CD20 monoclonal antibody therapy or stem cell transplant,[8] and thus antiviral prophylaxis is not currently recommended for such patients.[1] We found that a small proportion (8\%) of patients with past HBV infection receiving anticancer treatment that did not include anti-B cell therapy received antiviral therapy. Starting antiviral therapy without clear indications may subject patients to the 
burden of long-term therapy and financial costs without adding clinical benefit. Per ASCO guidelines[1] and expert opinion,[9] these patients could have close monitoring during cancer treatment, with antiviral therapy started at the earliest sign of HBV reactivation.

ASCO-supported guidance,[4] NCCN consensus,[2] and oncology experts[10] recommend that patients with cancer and HCV infection receive direct-acting antiviral therapy to eradicate HCV and decrease the risk of HCV-associated complications of anticancer treatment, including enhanced HCV replication and hepatitis flare, as well as the risk of secondary malignancies.[10, 4] Direct-acting antiviral therapy also prevents chronic hepatitis and progression to liver fibrosis or cirrhosis. Concomitant administration of anti-HCV therapy with anticancer therapy is generally not recommended by NCCN consensus,[2] although oncology experts offer alternative concomitant treatment strategies if patients can be closely monitored by a multidisciplinary team.[10] In our study, we found that only $6 \%$ of patients with HCV infection had received antivirals by 6 months.

NCCN consensus recommends that patients with cancer and HIV receive antiretroviral therapy before and during anticancer treatment in order to decrease the risk of infections and complications.[5] In our study, 10\% of patients with HIV had not received antiretroviral therapy by 6 months. We found high rates (up to $20 \%$ ) of co-infection with other viruses, supporting a comprehensive approach to screening for HBV, HCV, and HIV prior to the initiation of anticancer treatment.

Our study had several limitations. We examined outcomes in an observational cohort of patients from community-based oncology practices with a primary focus on seroprevalence of HBV, HCV, and HIV. Because this study examined only whether events had occurred by 6 months, we do not know if antiviral therapy was started to prevent or in response to hepatitis flare or other adverse liver outcomes. Antiviral therapy and clinical management were under the discretion of the providers; this approach mirrors actual practice, increasing the generalizability of our findings, but also resulted in a variety of viral management strategies. Also, since chronic HBV infection and HIV infection are relatively uncommon, our study included small numbers of patients with these viral infections, limiting our ability to draw strong conclusions about patterns of care for these patients. Another limitation is that study participants with co-infection were not treated separately in our analysis. Patients may have received more than one anticancer drug, and we did not separately analyze the myriad combinations. Anticancer treatments have evolved in recent years, and thus clinical outcomes could be different if the current study were repeated with the newer agents.

In summary, we found that antiviral therapy use often was not in agreement with national treatment guidelines. Our results suggest that providers caring for patients with viral infections receiving cancer treatment may need additional education and support. Future efforts focused on increasing awareness of guideline-recommended management strategies among oncology providers and increasing implementation of these strategies in clinical care could be warranted. Ongoing research priorities include ascertaining reactivation risk by specific anticancer drug, repeating this study in high-risk populations, developing longitudinal cohorts of cancer patients with viral infections, and prospective surveillance for hepatitis flares and their causes. Collaboration between medical providers who screen for viruses and those who manage the viral infections would be beneficial for patients with cancer. Such providers include specialists in oncology, infectious disease, hepatology, primary care, dissemination and implementation, decision support, clinical informatics, and electronic health records.

\section{Declarations}

Acknowledgements: We thank Stephanie Deming of the Research Medical Library at MD Anderson Cancer Center for editorial assistance. We are grateful to the study patients who generously gave their time and effort to this project.

Data availability: Data may be requested from the SWOG Cancer Research Network.

\section{Code availability: Not applicable}

Funding: Research reported in this publication was supported by the National Cancer Institute of the National Institutes of Health under award number UG1CA189974. The content is solely the responsibility of the authors and does not necessarily represent the official views of the National Institutes of Health.

\section{Competing interests:}

Jessica P. Hwang reports research funding to her institution from Merck. Rashmi Chugh reports research funding to her institution from AADi, Advenchen, Novartis, Epizyme, Lilly, Medivation, Mundipahrma, Glaxo Smith Kline, Janssen, Pfizer, Plexxikonn, Springworks, Qilu Puget Sound, and AstraZeneca. Rohit Loomba reports research funding to his institution from Arrowhead Pharmaceuticals,AstraZeneca, BoehringerIngelheim, Bristol-Myers Squibb, Eli Lilly, Galectin Therapeutics, Galmed Pharmaceuticals, Gilead, Intercept, Hanmi, Intercept, Inventiva, lonis, Janssen, Madrigal Pharmaceuticals, Merck, NGM Biopharmaceuticals, Novo Nordisk, Merck, Pfizer, Sonic Incytes, and Terns Pharmaceuticals; reports consulting roles at Aardvark Therapeutics, Altimmune, Anylam/Regeneron, Amgen, Arrowhead Pharmaceuticals, AstraZeneca, BristolMyer Squibb, CohBar, Eli Lilly, Galmed, Gilead, Glympse bio, Hightide, Inipharma, Intercept, Inventiva, Ionis, Janssen Inc., Madrigal, Metacrine, 
Inc., NGM Biopharmaceuticals, Novartis, Novo Nordisk, Merck, Pfizer, Sagimet, Theratechnologies, 89 bio, Terns Pharmaceuticals, and Viking Therapeutics; and is co-founder of LipoNexus. Scott D. Ramsey reports research funding to his institution from Bayer, Bristol-Myers Squibb, and Microsoft Corp and consulting roles at Bayer, Bristol-Myers Squibb, AstraZeneca, Merck, GRAIL, Pfizer, Seattle Genetics, Biovica, and Genentech. All other authors report no conflicts.

\section{Author contributions:}

Jessica P. Hwang: conceptualization, writing-original draft, writing-review and editing; Kathryn B. Arnold: formal analysis, conceptualization, writing-original draft, writing-review and editing; Joseph M. Unger: formal analysis, supervision, writing-review and editing; Rashmi Chugh: formal analysis, writing-review and editing; Monica A. Tincopa: formal analysis, writing-review and editing; Rohit Loomba: formal analysis, writing-review and editing; Dawn Hershman: formal analysis, writing-review and editing; Scott D. Ramsey: conceptualization, formal analysis, writing-review and editing, funding acquisition.

\section{Ethics approval:}

Institutional review board approval for the primary study, SWOG S1204, was provided by the Protocol Review Committee of the Cancer Therapy Evaluation Program on August 5, 2013.

\section{Consent to participate:}

Informed consent was obtained from all participants in SWOG S1204.

\section{Consent for publication:}

Not applicable

\section{References}

1. Hwang JP, Feld JJ, Hammond SP, Wang SH, Alston-Johnson DE, Cryer DR, Hershman DL, Loehrer AP, Sabichi AL, Symington BE, Terrault N, Wong ML, Somerfield MR, Artz AS (2020) Hepatitis B virus screening and management for patients with cancer prior to therapy: ASCO provisional clinical opinion update. J Clin Oncol Jco2001757. doi:10.1200/jco.20.01757

2. Baden LR, Swaminathan S, Angarone M, Blouin G, Camins BC, Casper C, Cooper B, Dubberke ER, Engemann AM, Freifeld AG, Greene JN, Ito JI, Kaul DR, Lustberg ME, Montoya JG, Rolston K, Satyanarayana G, Segal B, Seo SK, Shoham S, Taplitz R, Topal J, Wilson JW, Hoffmann KG, Smith C (2016) Prevention and Treatment of Cancer-Related Infections, Version 2.2016, NCCN Clinical Practice Guidelines in Oncology. J Natl Compr Canc Netw 14:882-913

3. Terrault NA, Lok ASF, McMahon BJ, Chang KM, Hwang JP, Jonas MM, Brown RS Jr, Bzowej NH, Wong JB (2018) Update on prevention, diagnosis, and treatment of chronic hepatitis B: AASLD 2018 hepatitis B guidance. Hepatology 67:1560-1599. doi:10.1002/hep.29800

4. Hwang JP, LoConte NK, Rice JP, Foxhall LE, Sturgis EM, Merrill JK, Torres HA, Bailey HH (2019) Oncologic Implications of Chronic Hepatitis C Virus Infection. J Oncol Pract 15:629-637. doi:10.1200/jop.19.00370

5. Reid E, Suneja G, Ambinder RF, Ard K, Baiocchi R, Barta SK, Carchman E, Cohen A, Gupta N, Johung KL, Klopp A, LaCasce AS, Lin C, Makarova-Rusher OV, Mehta A, Menon MP, Morgan D, Nathwani N, Noy A, Palella F, Ratner L, Rizza S, Rudek MA, Taylor J, Tomlinson B, Wang CJ, Dwyer MA, Freedman-Cass DA (2018) Cancer in People Living With HIV, Version 1.2018, NCCN Clinical Practice Guidelines in Oncology. J Natl Compr Canc Netw 16:986-1017. doi:10.6004/jnccn.2018.0066

6. Ramsey SD, Unger JM, Baker LH, Little RF, Loomba R, Hwang JP, Chugh R, Konerman MA, Arnold K, Menter AR, Thomas E, Michels RM, Jorgensen CW, Burton GV, Bhadkamkar NA, Hershman DL (2019) Prevalence of Hepatitis B Virus, Hepatitis C Virus, and HIV Infection Among Patients With Newly Diagnosed Cancer From Academic and Community Oncology Practices. JAMA oncology 5:497-505. doi:10.1001/jamaoncol.2018.6437

7. Loomba R, Rowley A, Wesley R, Liang TJ, Hoofnagle JH, Pucino F, Csako G (2008) Systematic review: The effect of preventive lamivudine on hepatitis $B$ reactivation during chemotherapy. Ann Intern Med 148:519-528

8. Paul S, Saxena A, Terrin N, Viveiros K, Balk EM, Wong JB (2016) Hepatitis B Virus Reactivation and Prophylaxis During Solid Tumor Chemotherapy: A Systematic Review and Meta-analysis. Ann Intern Med 164:30-40. doi:10.7326/m15-1121

9. Loomba R, Liang TJ (2017) Hepatitis B reactivation associated with immune suppressive and biological modifier therapies: current concepts, management strategies and future directions. Gastroenterology. doi:10.1053/j.gastro.2017.02.009

10. Torres HA, Shigle TL, Hammoudi N, Link JT, Samaniego F, Kaseb A, Mallet V (2017) The oncologic burden of hepatitis C virus infection: A clinical perspective. CA Cancer J Clin 67:411-431. doi:10.3322/caac.21403

Page 12/13 


\section{Figures}

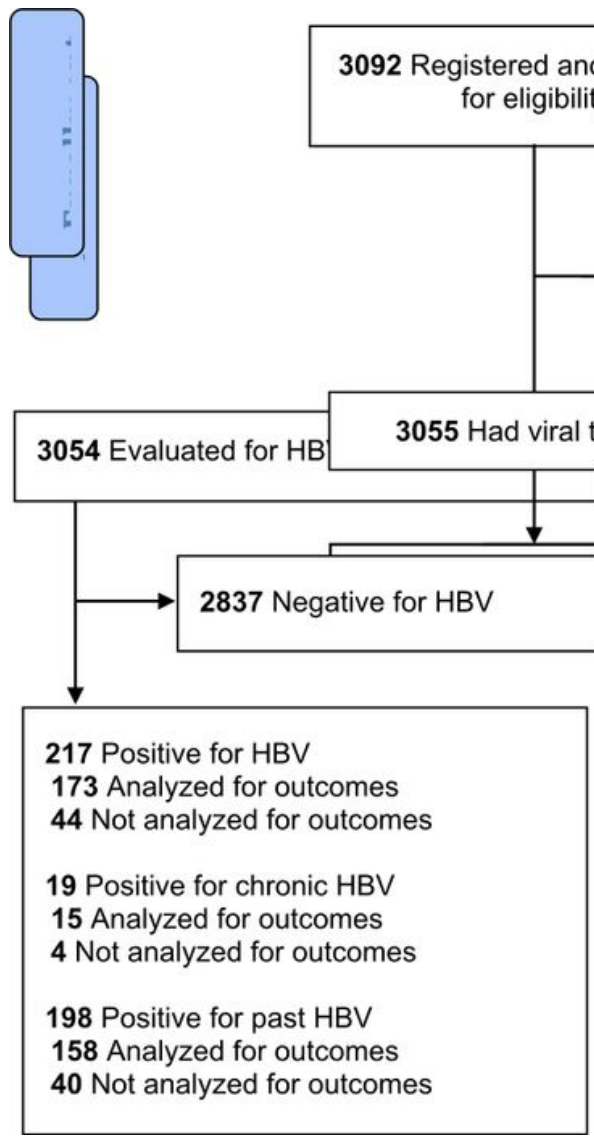

37 Excluded

34 Did not meet inclusion criteria

3 Declined to participate

\section{Figure 1}

Modified CONSORT flow diagram. HBV, hepatitis B virus; HCV, hepatitis C virus.

\section{Supplementary Files}

This is a list of supplementary files associated with this preprint. Click to download.

- OnlineOnlyAppendixTable013122.docx 